\title{
Sequential Vestibular Neuritis: Report of Four Cases and Literature Review
}

\author{
Francesco Comacchio ${ }^{1}$, Marta Mion $^{2}$, Enrico Armato ${ }^{3}$, and Andrea Castellucci ${ }^{4}$ \\ ${ }^{1}$ Division of Otolaryngology, Department of Neurosciences, Padova University, Padova, Italy \\ 2Division of Otolaryngology, Department of Specialistic Surgeries, Santa Maria della Misericordia Hospital, Rovigo, Italy \\ ${ }^{3}$ Division of Otolaryngology, Department of Surgery, SS. Giovanni e Paolo Hospital, Venezia, Italy \\ ${ }^{4}$ Division of Otolaryngology, Department of Surgery, Santa Maria Nuova Hospital, Reggio Emilia, Italy
}

\author{
Received June 1, 2020 \\ Revised September 30, 2020 \\ Accepted November 17, 2020 \\ Address for correspondence \\ Marta Mion, MD \\ Division of Otolaryngology, \\ Department of Specialistic Surgeries, \\ Santa Maria della Misericordia \\ Hospital, Viale Tre Martiri 140, \\ Rovigo 45100, Italy \\ Tel +39-425-3931 \\ Fax $+39-425-394715$ \\ E-mail med.mion@gmail.com
}

Background and Objectives: Bilateral sequential vestibular neuritis (BSVN) is a rare condition in which an inflammation or an ischemic damage of the vestibular nerve occurs bilaterally in a sequential pattern. We described four cases of BSVN. Subjects and Methods: Every patient underwent video-head impulse test during the first and the second episode of vestibular neuritis (VN), furthermore they have been studied with radiological imaging. Results: Contralateral VN occurred after a variable period from prior event. Vestibular function recovered from the first episode in one case. The other three patients developed contralateral VN. One case was due to a bilateral VN in association with a Ramsay-Hunt syndrome, in another patient clinical records strongly suggested an ischemic etiology, whereas in two cases aetiology remained uncertain. Two patients subsequently developed a benign paroxysmal positional vertigo involving the posterior canal on the side of the latest VN (Lindsay-Hemenway syndrome). Conclusions: Instrumental vestibular assessment represents a pivotal tool to confirm the diagnosis of $\mathrm{VN}$ and BSVN.

J Audiol Otol 2021;25(2):89-97

KEY WORDS: Benign paroxysmal positional vertigo · Facial paralysis · Head impulse test . Vestibular neuritis.

\section{Introduction}

Vestibular neuritis $(\mathrm{VN})$ is a peripheral vestibulopathy due to a sudden loss of vestibular function presenting with severe vertigo lasting over 24 hours, usually accompanied by nausea and vomiting. We recently demonstrated that acute vestibular loss could be caused by canalith jam [1,2], so we prefer to maintain the term "vestibular neuritis" to indicate an acute ischemic or viral vestibular loss. $\mathrm{VN}$ is characterized by spontaneous unidirectional nystagmus (SUN) beating towards the unaffected ear, positive bed-side head impulse test (HIT) on the horizontal plane and reduced or absent caloric response on the involved side. Auditory function and central nervous system (CNS) are generally spared. Complete symp-

This is an Open Access article distributed under the terms of the Creative Commons Attribution Non-Commercial License (https://creativecommons.org/licenses/by-nc/4.0/) which permits unrestricted non-commercial use, distribution, and reproduction in any medium, provided the original work is properly cited. toms relief usually occurs within six months from the onset $[3,4]$. Although the cause of $\mathrm{VN}$ is uncertain, it is thought to result from two different etiopathogenetic mechanisms: an inflammation involving the entire vestibular nerve or one of its divisions (presumably due to a viral reactivation) $[3,4]$ or an acute ischemia confined in the territory supplied by the anterior vestibular artery [5-7]. Nowadays, thanks to the recent introduction of fast devices for vestibular testing, information about the activity of peripheral sensors and afferents can be easily provided. The interpretation of data obtained combining the results of vestibular-evoked myogenic potentials (VEMPs) [8] and video-HIT [9] allows an accurate assessment of otolith and semicircular canals involvement, respectively, enabling the identification of specific patterns of lesion affecting labyrinthine end-organs or vestibular nerve divisions.

Bilateral sequential vestibular neuritis (BSVN) is a rare occurrence described in $1.9-5.3 \%$ of patients who previously developed a VN [10-12]. Contralateral recurrence of VN 
has been documented after a variable period from the prior event [10-14]. According to the English literature, few cases of BSVN [10-15] and two patients with simultaneous bilateral $\mathrm{VN}[16,17]$ have been reported so far. Given the rarity of reported cases, literature is still lacking in quantitative assessment of semicircular canals and otolith involvement. Here we describe the clinical presentation and the instrumental work-up in four cases of BSVN and discuss the possible underlying etiopathogenetic mechanisms.

\section{Subjects and Methods}

The four cases were selected according to typical characteristics of BSVN between 2012 and 2019, who visited "Padova University, Santa Maria della Misericordia Hospital, and Santa Maria Nuova Hospital". Every patient underwent video-HIT during the first and the second episode of VN, furthermore they have been studied with radiological imaging. The video-HIT has been performed with a camera assembled on a tripod connected to a computer that films the patient's face (Synapsys, Marseille, France). Where possible, VEMPs were evaluated in response to air-conducted sounds (tone bursts $500 \mathrm{~Hz}$, duration $8 \mathrm{~ms}$, intensity $120 \mathrm{~dB}$ SPL and stimulation rate $5 \mathrm{~Hz}$ ) using an Epic Plus system (Labat, Mestre, Italy) with a two channel averaging capacity, as reported by Rosengren, et al. [8]. Verbal informed consent for publication was obtained from every involved patient, agreeing with the written general consensus of the hospital admission that included scientific and bioethical research.

\section{Results}

All the cases are summarized in Table 1 according to the $\mathrm{SSC}$, the innervation and the vascularization involved during the first and the second episode of VN. The final outcome and added findings were also analyzed.

\section{Case 1}

A 61-year-old male was admitted to the ENT Unit complaining of acute rotatory vertigo without hearing impairment. Bed-side examination revealed right-beating SUN and positive HIT with leftward head rotations. Neurological evaluation was otherwise unremarkable and audiometry was within the age range. Contrast-enhanced brain CT scans and supraaortic trunks ultrasound were normal. The video-HIT showed reduced vestibulo-ocular reflex (VOR) gain for the left superior semicircular canal (SSC) and lateral semicircular canal (LSC). The patient was discharged with a diagnosis of left VN. Four years later, he experienced a second slight episode

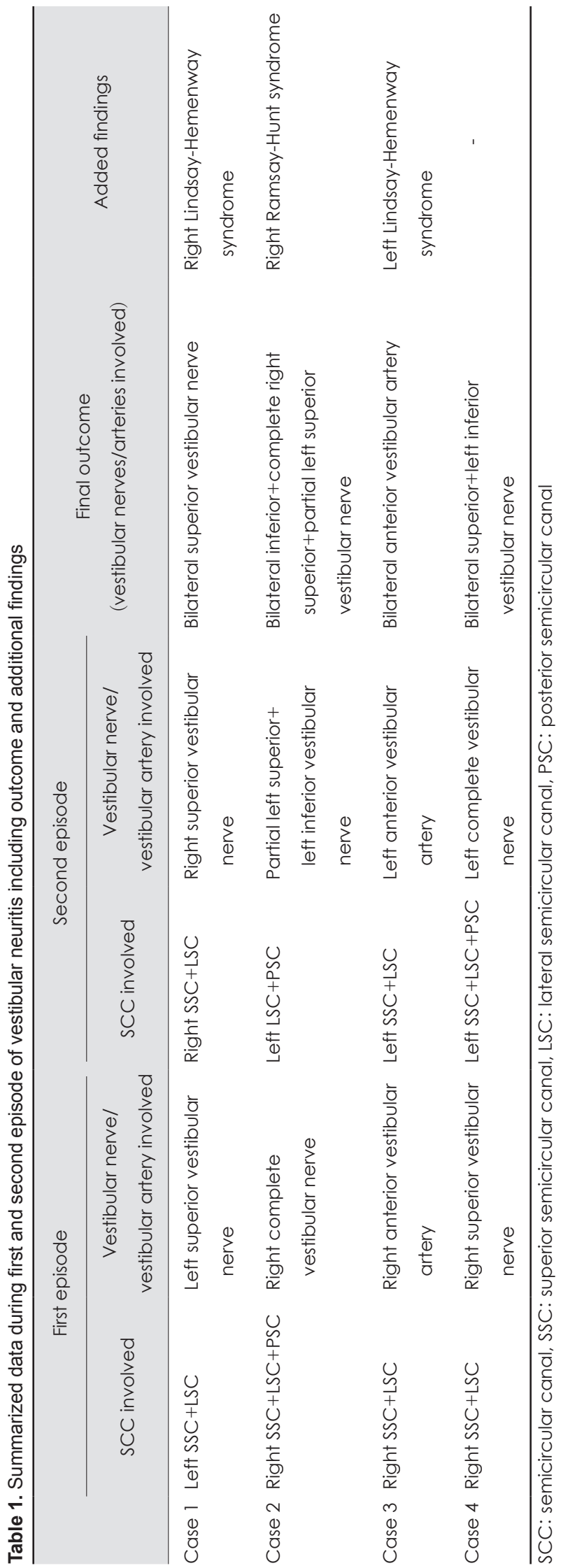

90 J Audiol Otol 2021;25(2):89-97 
Fig. 1. Video-HIT performed using an ICS VOG system (GN Otometrics, Taastrup, Denmark) with a highspeed head-mounted camera on goggles with velocity sensors. Mean value for the VOR gain (eye velocity/head velocity) is reported for each canal. This test demonstrates hypofunction of left SSC and LSC, and reduced gains for all right semicircular canals (with a slight functional impairment for the right PSC). LL: left lateral, RL: right lateral, LA: left anterior, RP: right posterior, LP: left posterior, RA: right anterior, HIT: head impulse test, VOR: vestibulo-ocular reflex, SSC: superior semicircular canal, LSC: lateral semicircular canal.

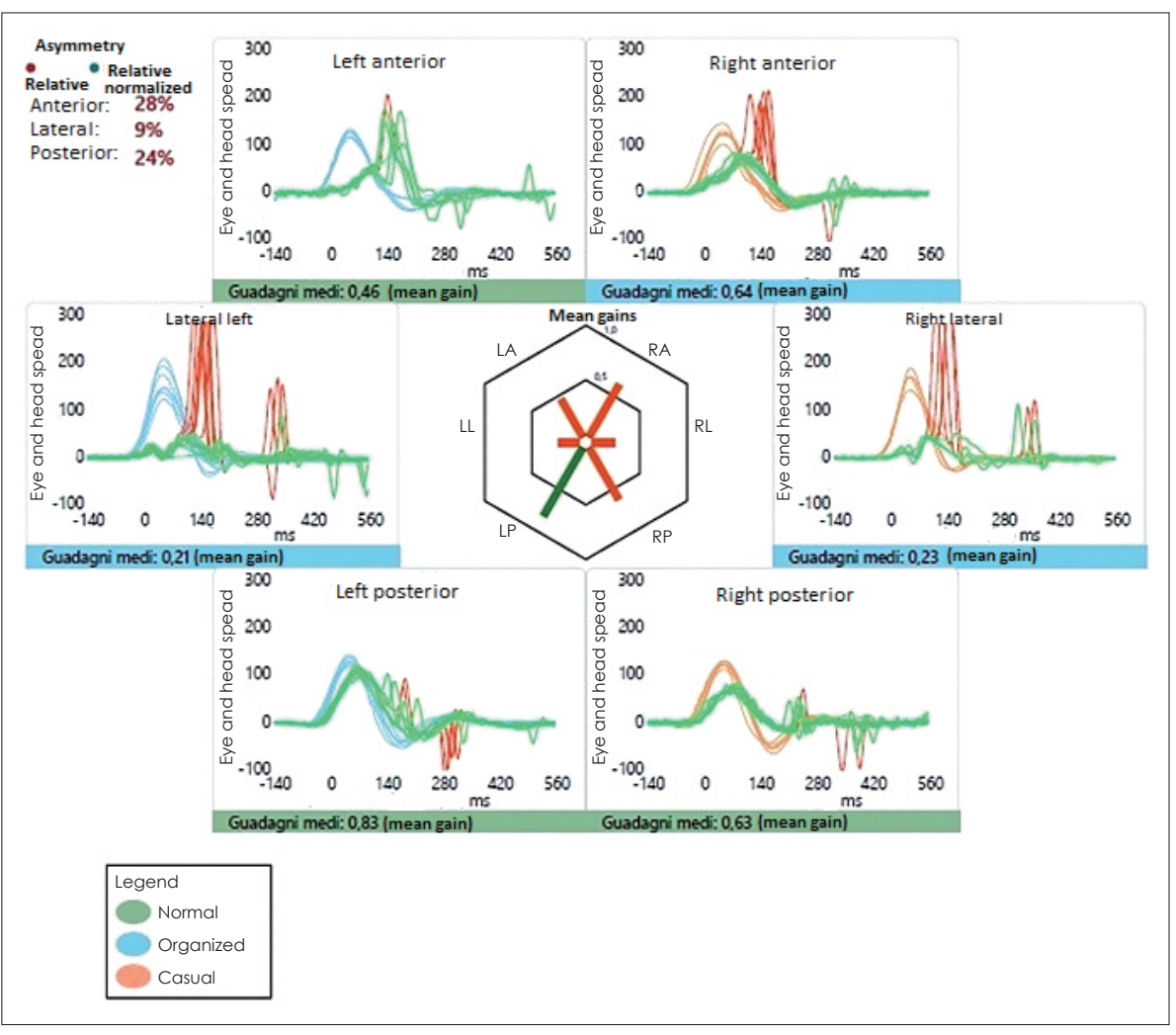

of acute vertigo and unsteadiness with left-beating SUN and refixation saccades after horizontal HIT in both directions. CNS involvement was ruled out by an extensive neurological assessment and a gadolinium-enhanced brain MRI. After corticosteroid treatment and resolution of symptoms, he developed positional vertigo few days later. Right Dix-Hallpike maneuver elicited paroxysmal up-beating/counter-clockwise nystagmus (with respect to the observer's point of view) consistent with benign paroxysmal positional vertigo (BPPV) due to right posterior semicircular canal (PSC) canalolithiasis. The patient was then successfully treated with multiple sessions of Epley particle repositioning maneuver (PRM). The video-HIT conducted after the second episode of VN and after the resolution of the BPPV demonstrated persistent left SSC and LSC hypofunction, newly reduced gains in the right SSC and LSC, and a slight functional impairment for the right PSC (Fig. 1). He developed severe imbalance, oscillopsia and blurred vision. He was suggested to follow a rehabilitation program focused on VOR and balance exercises.

\section{Case 2}

A 28-year-old male presented to the ENT Unit reporting right earache, dizziness, some vesicles on his right auricle and ipsilateral VII cranial nerve $(\mathrm{CN})$ palsy (V grade according to House Brackmann grading scale [18]). At the bed-side exami- nation he showed left-beating SUN and positive HIT on the right. Audiometric assessment showed normal hearing function (Fig. 2A). Fitzgerald-Hallpike bithermal caloric test (BCT) detected a complete right canal paresis (CP) (Fig. 2B). Gadolinium-enhanced brain MRI highlighted an increased enhancement in the intracanalicular tract of the right VIII CN (Fig. 2C, D). The patient was diagnosed with right RamsayHunt syndrome. Infusive therapy with glycerol, corticosteroids and antiviral drugs was administrated with improvement of vestibular symptoms while right facial palsy remained unchanged. At 8 days, the patient returned to the emergency department (ED) for sudden relapse of unsteadiness. He presented with right-beating SUN and positive horizontal HIT on both sides. Neither gaze-evoked nystagmus nor skew deviation could be detected. Ophthalmologic and neurological evaluations were unremarkable. Cerebrospinal fluid examination revealed a mild increase for white cells count without specific viral or bacterial contaminations. Extensive serological studies including auto-immunological panel ruled out specific immunological disorders. An additional gadolinium-enhanced brain MRI showed a newly enhanced signal in the left vestibular nerve (Fig. 2E, F). The video-HIT revealed bilateral vestibular loss with selective preservation of the left SSC function (Fig. 2G). After few days of infusive treatment, the patient was discharged with a diagnosis of Ramsay-Hunt syn- 


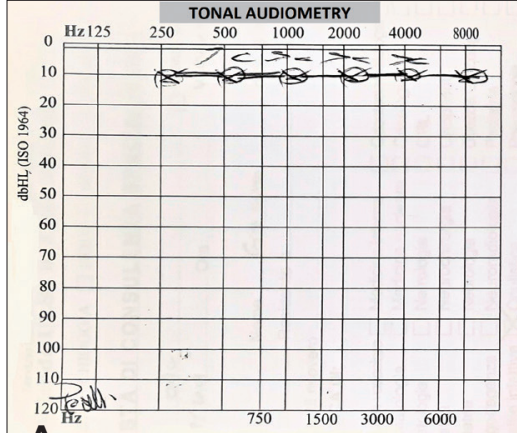

A
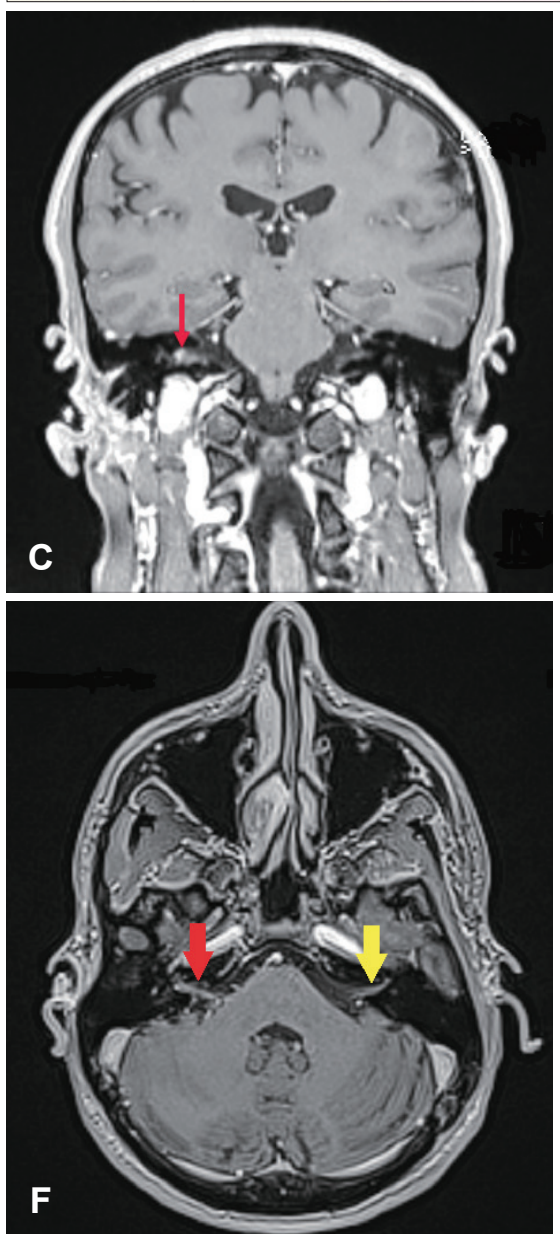
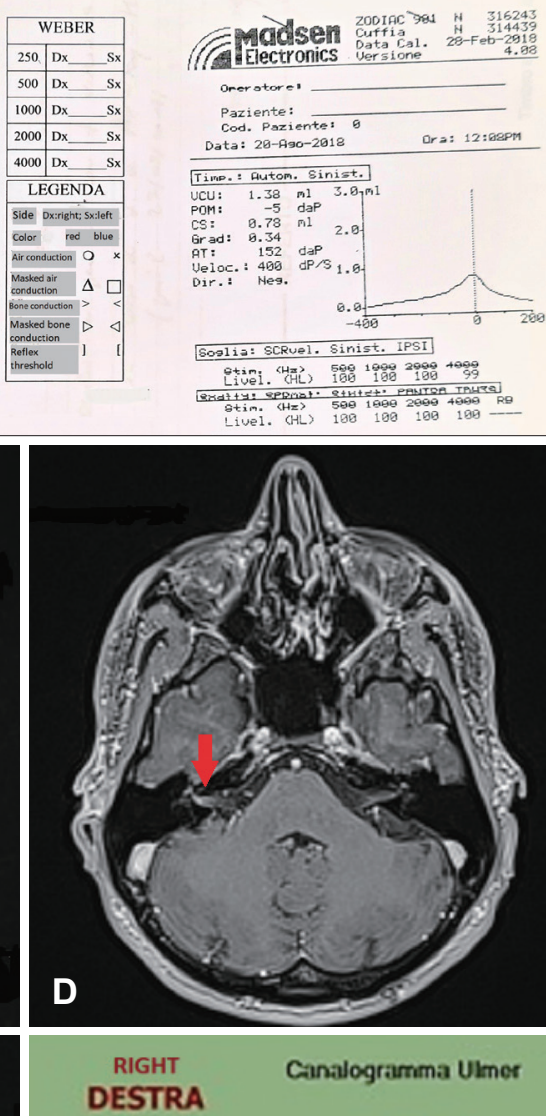
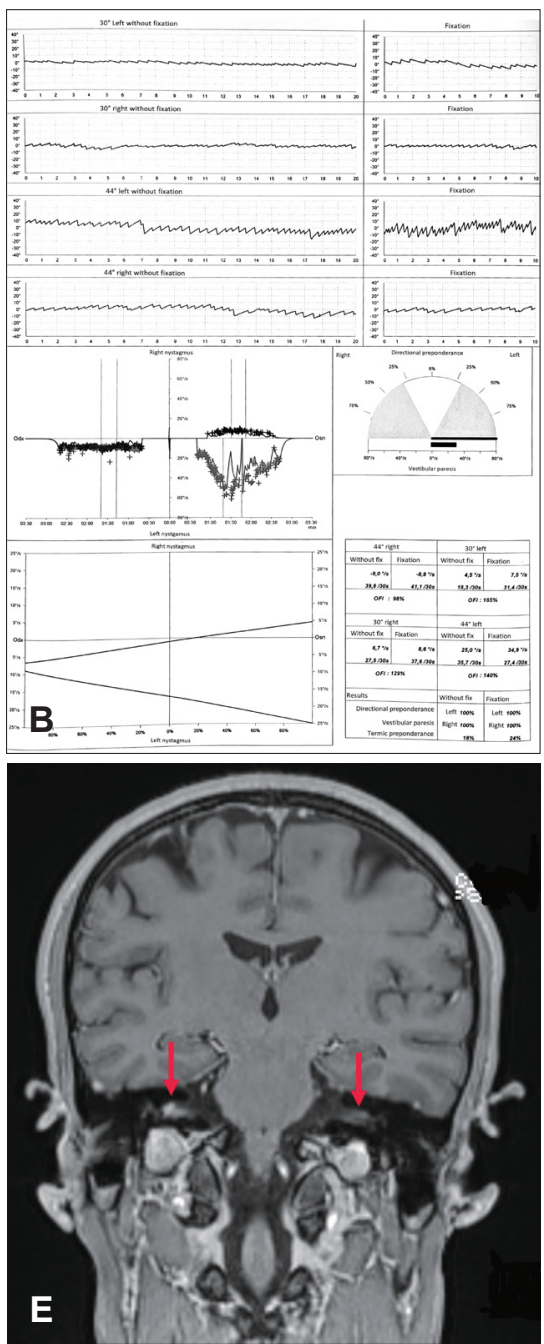

LEFT

SINISTRA

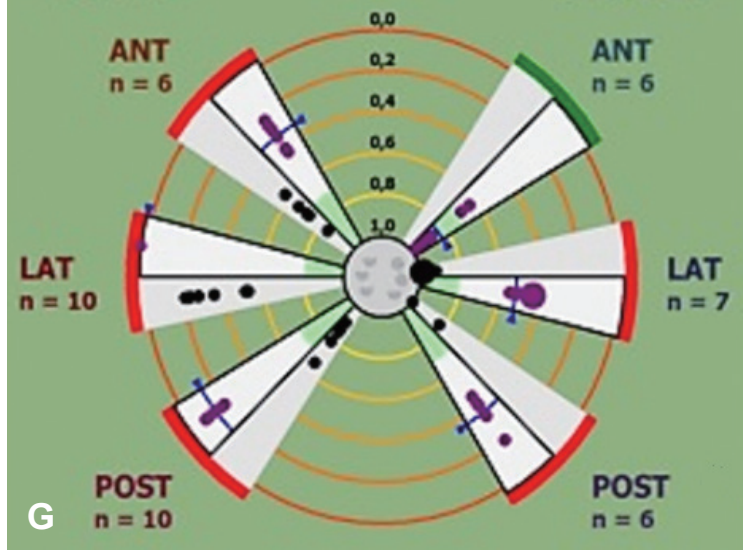

Fig. 2. Case 2 analyzed by audiometric assessment, bithermal caloric test, radiological imaging, and video-HIT (Synapsys, Marseille, France). A: Audiometric assessment shows normal hearing function. B: Fitzgerald-Hallpike bithermal caloric test shows a complete right canal paresis. C, D: Coronal and axial T1 sequences of the first gadolinium-enhanced brain MRI highlighting an increased enhancement in the intracanalicular tract of the right VIII CN (red arrow). E, F: Second coronal and axial T1 weighted post-contrast brain MRI showing persistent increased signal in the intracanalicular tract of the right VIII CN (red arrows) and newly slight enhancement in the left VIII CN (yellow arrow). G: The value of VOR gain for each impulse is graphically expressed as a dot within concentric circle representing VOR gain ranges. A complete functional impairment for all right semicircular canals and for left LSC and PSC can be observed, whereas left SSC function is selectively spared. OFI: ocular fixation index, ANT: anterior, LAT: lateral, POST: posterior, HIT: head impulse test, CN: cranial nerve, SSC: superior semicircular canal, VOR: vestibulo-ocular reflex, LSC: lateral semicircular canal, PSC: posterior semicircular canal. 
drome with peripheral involvement of right VII CN and vestibular nerves on both sides. On one-month follow-up, he did not show any functional improvement neither in right facial nor in semicircular canals function on both sides at the videoHIT. Though symptom-free at rest and low-velocity walking, he reported disabling oscillopsia during speed walking or running, so he was addressed to an intensive vestibular rehabilitation therapy.

\section{Case 3}

A 63-year-old male was admitted to the ED for acute onset of vertigo. Bed-side examination revealed left-beating SUN and refixation saccades after right HIT. Audiometry assessed a symmetrical high-frequency sensorineural hearing loss con- sistent with the patient's age. Contrast-enhanced brain CT scan showed signs of chronic vascular disease and Doppler ultrasonography of the supra-aortic trunks revealed a $40 \%$ arterial lumen stenosis at carotid bifurcation bilaterally. Neurological examination was uneventful. The patient was discharged for right VN. He returned to our attention four months later for recurrence of slight vertigo and unsteadiness. Right-beating SUN was registered with video-oculography (VOG). The videoHIT showed bilateral hypofunction of the LSC and SSC (Fig. $3 \mathrm{~A})$. At 1 month from the onset, only a slight pure up-beating nystagmus increased by the skull vibration test could be detected by VOG in supine position. Cervical VEMPs for air-conducted sounds showed normal amplitudes on both sides, while air-conducted ocular VEMPs were bilaterally absent (Fig. 3B).
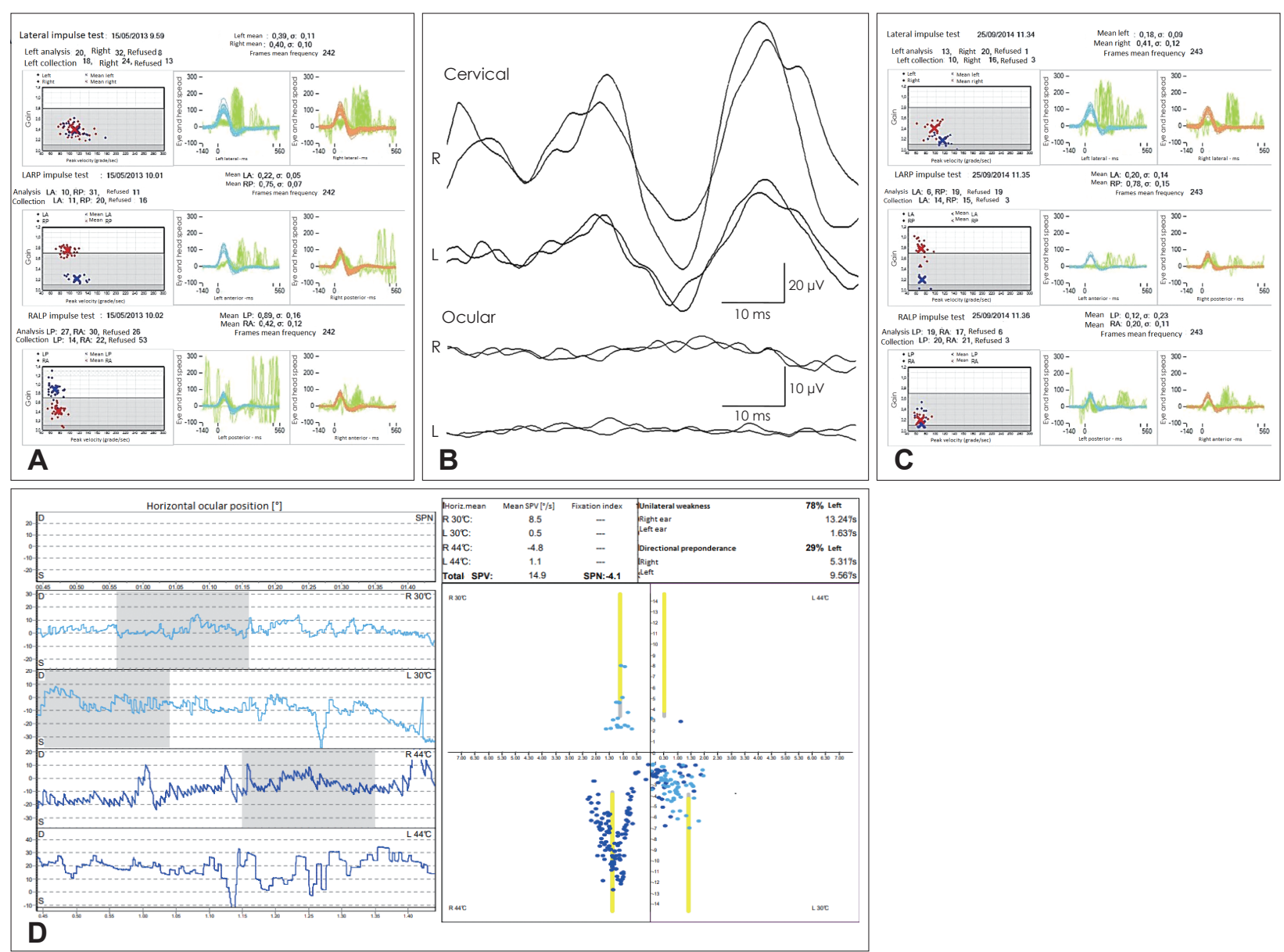

Fig. 3. Case 3 analyzed by video-HIT, VEMPs, and bithermal caloric test. A: Video-HIT performed using an ICS VOG system (GN Otometrics, Taastrup, Denmark) showing bilateral hypofunction of the LSC and the SSC. B: Cervical (above) and ocular (below) VEMPs. Cervical VEMPs amplitudes are slightly reduced on the left though within normality ranges (right: $50.4 \mu \mathrm{V}$, left: $28.6 \mu \mathrm{V}$, asymmetry ratio: $28 \%$ ), whereas ocular VEMPs are bilaterally absent. C: Video-HIT showing a complete deficit for all left semicircular canals and an impairment of the right LSC and SSC. Mean value of the VOR gain (eye velocity/head velocity) and corresponding standard deviation $(\sigma)$ is reported for each canal. D: BTC results after the second episode of VN documenting bilateral vestibular hypofunction with left CP of $78 \%$. LARP: left anterior-right posterior, RALP: right anterior-left posterior, R: right, L: left, SPV: slow phase velocity, HIT: head impulse test, VEMPs: vestibular-evoked myogenic potentials, LA: left anterior, RP: right posterior, LP: left posterior, RA: right anterior, VOR: vestibulo-ocular reflex, LSC: lateral semicircular canal, SSC: superior semicircular canal, BTC: bithermal caloric (test), VN: vestibular neuritis, CP: canal paresis. 
Temporal bone CT scan was unremarkable, while brain MRI showed wide multifocal ischemic leukoencephalopathy with mild enlargement of brain cerebrospinal fluid spaces. The patient was treated with statins and anticoagulant medications because of his cardiovascular comorbidities and sent to a vestibular rehabilitation program for severe imbalance and oscillopsia. The same patient returned to the ED 17 months later for sudden onset of positional vertigo. Left Dix-Hallpike maneuver elicited paroxysmal up-beating/clockwise nystagmus (with respect to the observer's point of view) consistent with left PSC-BPPV. Several Epley PRMs were needed to treat the patient effectively. At 10 days, the video-HIT showed reduced gains in all left semicircular canal planes and for the right LSC and SSC (Fig. 3C). BCT demonstrated bilateral vestibular hypofunction with left CP of $78 \%$ according to Jongkees formula (Fig. 3D).

\section{Case 4}

A 36-year-old female was admitted to the ENT Unit in 2004 for an acute vestibular syndrome without hearing impairment or CNS symptoms. A slight left-beating SUN and positive HIT for rightward head rotations could be detected at the bedside examination. The video-HIT showed a selective VOR impairment for the right SSC and LSC. Audiometric assessment, neurological evaluation and gadolinium-enhanced brain MRI were unremarkable. BCT revealed a severe right CP (80\%). She was submitted to infusive steroids therapy for relief of symptoms and discharged with a diagnosis of right VN. At 7 days, the video-HIT documented a normalization of VOR gain for right SSC and LSC. Four years after the first event, she experienced a second episode of long-lasting rotatory vertigo with similar characteristics to the previous manifestation. VOG detected a right-beating SUN, whereas neurological assessment and brain MRI excluded CNS involvement. The videoHIT showed reduced gains in all left semicircular canal planes (Fig. 4). On six-month follow-up, the video-HIT did not show any sign of functional recovery of the left side. Nevertheless, the patient returned to fulltime work and daily activity.

\section{Discussion}

BSVN is a rare condition. According to literature, only 1.9$5.3 \%$ of patients with VN develops a bilateral involvement with different onset time for each affected ear [10-12], whereas a relapse rate on the ipsilateral side has been documented in up to $16.7 \%$ of patients with VN $[19,20]$. In this study, we found four cases of BSVN ( 3 males and 1 female, mean age $47.0 \pm 17.6$ years, range $28-63$ years). All patients fulfilled criteria for $\mathrm{VN}$ in each occasion based on clinical diagnosis on prolonged vertigo, horizontal SUN, positive HIT and lack

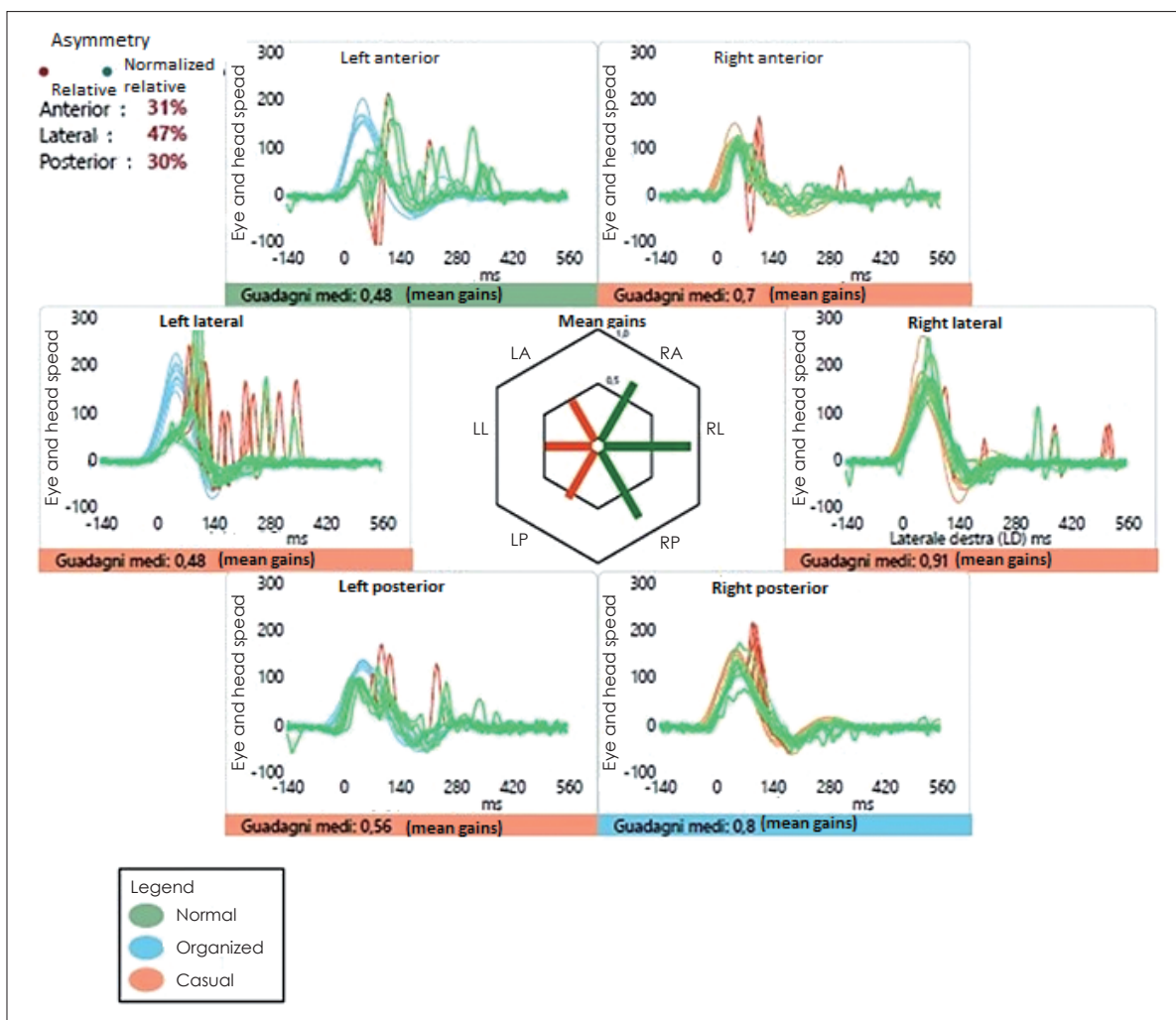

Fig. 4. Video-HIT performed using an ICS VOG system (GN Otometrics, Taastrup, Denmark) showing reduced values of VOR gain for all left semicircular canals. Mean value for the VOR gain (eye velocity/head velocity) is reported for each canal. HIT: head impulse test, LL: left lateral, RL: right lateral, LA: left anterior, RP: right posterior, LP: left posterior, RA: right anterior, VOR: vestibulo-ocular reflex. 
of auditory system or CNS involvement [3,4]. In accordance with literature, the first manifestation appeared variable period (ranging from few days to four years) before involvement of the second ear [10-15] and recurrence was generally less symptomatic compared to the first episode [11,15]. Since vestibular hypofunction can either restore or persist after VN $[19,20]$, this different behavior may explain the different symptomatology reported by the same patient during each episode of VN. In fact, while in case of functional recovery from the first $\mathrm{VN}$ contralateral recurrence should result in the same severity of symptoms as the prior manifestation (as for Case 4 in our report), if vestibular loss persists at the time of the second event both labyrinths lack in function and gait ataxia, unsteadiness and oscillopsia should prevail (as for the other 3 patients). BSVN can indeed account for a small percentage of cases with bilateral vestibular loss, condition generally associated with ototoxic drugs, autoimmune inner ear diseases or bilateral Ménière's disease [21]. All three patients from our report who did not restore vestibular function from the first episode developed spontaneous horizontal nystagmus beating towards this side during contralateral VN, despite persistent vestibular hypofunction. This phenomenon was first described by Bechterew after sequential disruption of both labyrinths in animal experiments, and it is thought to be due to the action of compensation mechanisms used by CNS after acute onset of vestibular asymmetry [22] rather than to residual vestibular activity on the side of the first event.

The aetiology of $\mathrm{VN}$ is still a matter of debate. A viral genesis is strongly suspected due to its pathological similarity to reported cases of herpes zoster oticus and frequent connections between $\mathrm{VN}$ and previous infections of the upper respiratory tract. The superior division of the vestibular nerve, that innervates the SSC, the LSC and the utricle (i.e., superior labyrinth), has demonstrated to be more frequently involved than the inferior branch collecting PSC and saccular afferents (inferior labyrinth) [23]. Possible explanations to this selective vulnerability rely on the different anatomy of the bony channels of the vestibular nerves and on a selective tropism for viral agents that leads the superior division to be more susceptible to injuries [23-25]. Another possible etiopathogenetic factor for $\mathrm{VN}$ is an ischemic damage in the labyrinth. In support of this assumption, a significantly higher prevalence of cardiovascular risk factors was found in patients with $\mathrm{VN}$ in comparison to the general population [26] and unilateral CP on calorics was detected in approximately half of patients with vertebrobasilar insufficiency [6]. Vascular damage in the inner ear could reflect either a more extensive deficiency in the posterior fossa perfusion or a more selective alteration of the microcirculation in the peripheral receptor, generally lacking in collateralizations $[6,7]$. Based on anatomical studies, the anterior vestibular artery is the smallest terminal branch of the internal auditory artery and is equipped by little collateral circulation [27], representing the most likely candidate site accounting for isolated vertigo of vascular origin. As it supplies mostly the same structures innervated by the superior vestibular nerve [7,27], an ischemic damage at this level is hardly distinguishable from superior VN. In both cases, PSC and most saccular function is generally preserved. Given the multifocal ischemic leukoencephalopathy confirmed by MRI in Case 3, we hypothesized a selective sequential microischemia involving both anterior vestibular arteries to be the most likely mechanism accounting for BSVN in this patient. Conversely, imaging showed a bilateral sequential neural involvement in Case 2 , confirming a bilateral VN in this case. In fact, Ramsay-Hunt syndrome is a well-known condition generally attributed to a reactivation of herpes zoster virus (HZV) at the geniculate ganglion, and it is characterized by severe ear pain, vesicular eruptions within the external auditory canal and peripheral VII $\mathrm{CN}$ paralysis. Other CN such as V, VIII, IX, XI, and XII could be involved. Connections between facial and vestibular nerves within the internal auditory canal can explain the association of VN with Ramsay-Hunt syndrome and other unexpected vestibular disturbances associated with facial paralysis $[23,28]$. For the other two patients in our report, neither history nor imaging suggested certain or likely causes for BSVN.

In agreement with literature concerning $\mathrm{VN}[4,23,25]$, the majority of overall sequential acute episodes in our report documented a sure involvement of the superior vestibular nerve basing on video-HIT data. In one case a VN due to HZV reactivation selectively spared the SSC afferents though involving both vestibular nerve divisions. Only in Case 3 utricular and saccular function were tested with ocular and cervical-VEMPs, respectively, confirming a selective functional preservation of the inferior vestibular nerve. Residual activity for both PSC could likely account for the slight pure up-beating nystagmus increased by skull vibration that was detected in this patient after the second VN. In fact, both SSC and LSC hypofunction likely resulted in an unopposed excitation of both PSC with mastoid vibration. The resulting symmetrical and selective activation of both active PSC could lead to the onset of a pure up-beating nystagmus [16].

Utricular injury and residual activity in the PSC represent the pathophysiological background for the so-called LindsayHemenway syndrome [5]. This condition accounts for PSC$\mathrm{BPPV}$ following a VN and it is thought to develop in case of superior $\mathrm{VN}$ where a utricular damage results in otoliths release into a functionally preserved PSC. Though originally attributed to an ischemic insult in the territory supplied by 
the anterior vestibular artery [5], it has been also described in $9.8-15.3 \%$ of $\mathrm{VN}$, likely as a consequence of a direct macular damage or nerve deafferentation $[11,15,18]$. Two patients from our report developed BPPV following a VN (i.e., $25 \%$ of overall sequential acute episodes). In both cases, BPPV occurred after the latest manifestation and more than one PRM was needed to treat positional vertigo, as reported in literature $[15,18]$. While in Case 1 aetiology could not be ascertained and both superior $\mathrm{VN}$ and labyrinthine infarction could be hypothesized, anterior vestibular artery ischemia could likely account for the clinical scenario in Case 3. It is interesting to notice that a mild functional impairment for the PSC could be found in both cases after successful PRM for BPPV. A reduction of VOR gain for the PSC may reflect a progression in labyrinthine damage, either due to microvascular disease in small arterial branches supplying PSC or to posterior ampulla deafferentation. Since observing a PSC-BPPV after a VN implies a functional preservation of the PSC ampulla, it is possible to assume that, despite instrumental findings, ampullary function could be enough preserved to allow a severe canalolithiasis to occur. Alternatively, any selective lesion to irregular afferents could hypothetically result in a dissociation between high- and low-frequency domains, impeding PSC to respond to high-frequency stimuli (video-HIT), whereas preserved regular fibers would allow the cupula to be stimulated by low-frequency stimuli (free-floating otoconia) [29]. On the other hand, slightly reduced VOR-gain values with small covert saccades of the PSC (functionally coupled with contralateral affected SSC) in cases with selective involvement of superior vestibular nerve or superior labyrinthine ischemia are also in line with studies on contralesional canal activity following VN showing, on average, mild impaired activity in the healthy side. In fact, abnormalities detected on contralesional PSC plots may be likely explained by severely reduced activity for injured SSC. It is still unclear whether peripheral phenomena (i.e., functional loss of the "push-pull" mechanism) or central compensation (i.e., cerebellar "shut-down") may account for these findings. Probably, whereas the first theory may explain reduced PSC VOR-gain in acute stage, the latter phenomenon may likely explain PSC abnormalities in those patients with long-lasting symptoms.

Nevertheless, according to our findings, BPPV seems to occur more frequently in BSVN than in isolated cases of VN (50\% compared to $9.8-15.3 \%$, respectively). These findings can reflect a tendency for inner ears of patient with BSVN to feature an extremely susceptible neural or vascular system.

In conclusion, we described four cases of BSVN and discussed the significance of our findings with respect to the literature review. Clinicians should keep in mind that both VN and labyrinthine ischemia can occur bilaterally in a sequential pattern, although rarely and in a small proportion of patients compared with unilateral VN. Brain imaging should always be given as it may help in the identification of specific causes for BSVN besides excluding CNS involvement. Video-HIT and VEMPs represent a noninvasive test battery offering a comprehensive assessment of receptors and afferents involvement. As they are rapid tools for vestibular testing, we suggest clinicians using them to follow the vestibular function of patients with unilateral $\mathrm{VN}$ or BSVN in order to evaluate functional recovery over time to predict possible recurrences or late onset of BPPV.

\section{Acknowledgments \\ None}

\section{Conflicts of interest}

The authors have no financial conflicts of interest.

\section{Author Contributions}

Conceptualization: Francesco Comacchio. Data curation: Enrico Armato and Andrea Castellucci. Formal analysis: Francesco Comacchio and Enrico Armato. Investigation: all authors. Methodology: Francesco Comacchio and Enrico Armato. Project administration: Francesco Comacchio. Supervision: all authors. Validation: all authors. Visualization: all authors. Writing — original draft: Marta Mion. Writingreview \& editing: Marta Mion, Andrea Castellucci, and Francesco Comacchio. Approval of final manuscript: all authors.

\section{ORCID iDs}

Francesco Comacchio https://orcid.org/0000-0001-5913-1484

Marta Mion

Enrico Armato https://orcid.org/0000-0002-2927-5871

Andrea Castellucci https://orcid.org/0000-0003-2874-5672

\section{REFERENCES}

1) Comacchio F, Poletto E, Mion M. Spontaneous canalith jam and apogeotropic horizontal canal benign paroxysmal positional vertigo: considerations on a particular case mimicking an acute vestibular deficit. Otol Neurotol 2018;39:e843-8.

2) Castellucci A, Malara P, Brandolini C, Del Vecchio V, Giordano D, Ghidini A, et al. Isolated horizontal canal hypofunction differentiating a canalith jam from an acute peripheral vestibular loss. Am J Otolaryngol 2019;40:319-22.

3) Coats AC. Vestibular neuronitis. Trans Am Acad Ophthalmol Otolaryngol 1969;73:395-408.

4) Schuknecht HF, Kitamura K. Second Louis H. Clerf lecture. Vestibular neuritis. Ann Otol Rhinol Laryngol Suppl 1981;90(1 Pt 2):119.

5) Lindsay JR, Hemenway WG. Postural vertigo due to unilateral sudden partial loss of vestibular function. Ann Otol Rhinol Laryngol 1956;65:692-706.

6) Grad A, Baloh RW. Vertigo of vascular origin. Clinical and electronystagmographic features in 84 cases. Arch Neurol 1989;46:281-4.

7) Kim JS, Lee H. Inner ear dysfunction due to vertebrobasilar ischemic stroke. Semin Neurol 2009;29:534-40.

8) Rosengren SM, Welgampola MS, Colebatch JG. Vestibular evoked myogenic potentials: past, present and future. Clin Neurophysiol 2010; 
121:636-51

9) Halmagyi GM, Chen L, MacDougall HG, Weber KP, McGarvie LA, Curthoys IS. The video head impulse test. Front Neurol 2017;8:258.

10) Ogata Y, Sekitani T, Shimogori H, Ikeda T. Bilateral vestibular neuronitis. Acta Otolaryngol Suppl 1993;503:57-60.

11) Huppert D, Strupp M, Theil D, Glaser M, Brandt T. Low recurrence rate of vestibular neuritis: a long-term follow-up. Neurology 2006; 67:1870-1.

12) Sekitani $T$, Imate $Y$, Noguchi $T$, Inokuma $T$. Vestibular neuronitis: epidemiological survey by questionnaire in Japan. Acta Otolaryngol Suppl 1993;503:9-12.

13) Schuknecht HF, Witt RL. Acute bilateral sequential vestibular neuritis. Am J Otolaryngol 1985;6:255-7.

14) Young AS, Taylor RL, McGarvie LA, Halmagyi GM, Welgampola MS. Bilateral sequential peripheral vestibulopathy. Neurology 2016; 86:1454-6.

15) Kim YH, Kim KS, Kim KJ, Choi H, Choi JS, Hwang IK. Recurrence of vertigo in patients with vestibular neuritis. Acta Otolaryngol 2011;131:1172-7.

16) Yacovino DA, Finlay JB, Urbina Jaimes VN, Verdecchia DH, Schubert MC. Acute bilateral superior branch vestibular neuropathy. Front Neurol 2018;9:353.

17) Ichijo K, Kinoshita M, Fujimoto C, Uranaka T, Kikkawa YS, Sugasawa K, et al. Acute bilateral vestibulopathy with simultaneous involvement of both superior and inferior vestibular nerves. Auris Nasus Larynx 2020;47:905-8.

18) House JW, Brackmann DE. Facial nerve grading system. Otolaryngol Head Neck Surg 1985;93:146-7.

19) Bergenius J, Perols O. Vestibular neuritis: a follow-up study. Acta Otolaryngol 1999;119:895-9.
20) Mandalà M, Santoro GP, Awrey J, Nuti D. Vestibular neuritis: recurrence and incidence of secondary benign paroxysmal positional vertigo. Acta Otolaryngol 2010;130:565-7.

21) Hain TC, Cherchi M, Yacovino DA. Bilateral vestibular loss. Semin Neurol 2013;33:195-203.

22) Katsarkas A, Galiana HL. Bechterew's phenomenon in humans. A new explanation. Acta Otolaryngol Suppl 1984;406:95-100.

23) Himmelein S, Lindemann A, Sinicina I, Horn AKE, Brandt T, Strupp M, et al. Differential involvement during latent herpes simplex virus 1 infection of the superior and inferior divisions of the vestibular ganglia: implications for vestibular neuritis. J Virol 2017;91: e00331-17.

24) Proctor L, Perlman H, Lindsay J, Matz G. Acute vestibular paralysis in herpes zoster oticus. Ann Otol Rhinol Laryngol 1979;88(3 Pt 1): 303-10.

25) Gianoli G, Goebel J, Mowry S, Poomipannit P. Anatomic differences in the lateral vestibular nerve channels and their implications in vestibular neuritis. Otol Neurotol 2005;26:489-94.

26) Oron Y, Shemesh S, Shushan S, Cinamon U, Goldfarb A, Dabby R, et al. Cardiovascular risk factors among patients with vestibular neuritis. Ann Otol Rhinol Laryngol 2017;126:597-601.

27) Mazzoni A. The vascular anatomy of the vestibular labyrinth in man. Acta Otolaryngol Suppl 1990;472:1-83.

28) Ozdoğmuş O, Sezen O, Kubilay U, Saka E, Duman U, San T, et al. Connections between the facial, vestibular and cochlear nerve bundles within the internal auditory canal. J Anat 2004;205:65-75.

29) Casani AP, Cerchiai N, Navari E. Paroxysmal positional vertigo despite complete vestibular impairment: the role of instrumental assessment. Acta Otorhinolaryngol Ital 2018;38:563-8. 